\title{
Psychometric evaluation of the Pinocchio Illusion Questionnaire
}

\author{
John R. Purcell ${ }^{1,2}$ • John Chen ${ }^{1}$ • Alexandra B. Moussa-Tooks ${ }^{1,2}$ - William P. Hetrick ${ }^{1,2,3}$ \\ Published online: 17 March 2020 \\ (C) The Psychonomic Society, Inc. 2020
}

\begin{abstract}
Perceived nose elongation resulting from vibratory stimulation to the bicep brachii tendon in the absence of visual input while the finger is touching the nose, known as the Pinocchio Illusion (PI), is used to investigate how afferent signals can contribute to aberrant top-down perception of body representation. The Pinocchio Illusion Questionnaire (PIQ) was developed to empirically quantify PI perception, allowing for external validation of the PI with psychologically relevant phenomenon. The current study $(N=60)$ examined the PIQ's test-retest reliability, internal consistency, factor structure, and correlations with self-reported interoceptive awareness and schizotypal traits. The PIQ demonstrated strong test-retest reliability and internal validity; however, a Principal Component Analysis did not yield a latent variable structure that distinguished PI-specific perceptual aberrations from unrelated or contradictory perceptual experiences. Additionally, decreased reports of PI-specific perceptual aberrations during two elicitations of the PI on the PIQ's open-ended free-response section (percent of sample endorsement $=5 \%$ (first elicitation); $8.3 \%$ (second elicitation)) compared to its 11-item section (endorsement of PI-specific items ranging 30-53.33\% (first)]; 31.67$46.67 \%$ (second)) suggest that these responses may be heavily influenced by demand characteristics rather than accurately capturing PI perception. Therefore, further psychometric development of the PIQ and standardization of procedures to elicit the illusion are recommended.
\end{abstract}

Keywords Pinocchio Illusion $\cdot$ Proprioception $\cdot$ Haptics $\cdot$ Embodied perception $\cdot$ Bodily illusion $\cdot$ Schizotypy

\section{Introduction}

Sensory illusions provide unique insights into interactions among visual, haptic, and proprioceptive systems that map bodily boundaries and relative locations of limbs in space (Proske \& Gandevia, 2012). The Pinocchio Illusion (PI), a perceptual illusion of nose elongation resulting from vibratory stimulation to the bicep brachii tendon in the absence of visual input while the finger is touching the nose, specifically

John R. Purcell and John Chen share first authorship.

Electronic supplementary material The online version of this article (https://doi.org/10.3758/s13414-020-02011-4) contains supplementary material, which is available to authorized users.

William P. Hetrick

whetrick@indiana.edu

1 Department of Psychological and Brain Sciences, Indiana University, 1101 E. 10th St., Bloomington, IN 47408, USA

2 Program in Neuroscience, Indiana University, Bloomington, IN, USA

3 Department of Psychiatry, Indiana University School of Medicine, Indianapolis, IN, USA demonstrates how manipulation of somatosensory and proprioceptive afferents can modulate experiences of bodily shape perception via top-down processing (Burrack \& Brugger, 2005; Lackner, 1988). Developments in empirical elicitation and measurement of the PI, and recent evaluation of aberrant bodily perception more broadly, in clinical disorders (e.g., schizophrenia/schizotypy; Benson \& Park, 2019; Michael \& Park, 2016) has resulted in a resurgence of interest in this perceptual illusion.

PI perception is hypothesized to result from proprioceptive afferent signals sent from nerve endings in muscle spindles triggered by vibratory stimulation, resulting in the perception of movement and/or altered bodily position (i.e., "kinesthesia" and "position sense"; Goodwin, McCloskey, \& Matthews, 1972; for review see Proske \& Gandevia, 2012). Previous work has found that the perception of movement may be more attributable to primary nerve endings that detect changes in muscle length caused by vibration frequency, whereas perception of position may be impacted by primary and secondary nerve endings, as well as receptors in joints and tendons (Roll \& Vedel, 1982). While these two perceptual processes may partially share sensory pathways, muscle vibration at lower frequencies and larger amplitudes has resulted in altered position sense without reported changes in movement (McCloskey, 1973). 
Over time, muscle spindle nerve signals, resulting from general movement, contribute to the construction and maintenance of internal models of typical position sense, which then serve as a priori predictions for future positional experiences. When these well-trained models are presented with conflicting haptic (finger-nose) and proprioceptive (muscle spindle) inputs without reconciliatory visual input (Lackner, 1988; Lackner \& Taublieb, 1984), illusory post hoc PI perceptions arise including nose-elongation, arm elongation, or even a disconnection between arm, nose, hand, or finger.

Unlike some proprioceptive illusions, the PI is especially difficult to measure as it is a phenomenological experience. This seemingly limits quantification to subjective, selfreported sensations rather than precise physical measurements. Nonetheless, studies of other body-perception illusions have found relationships between self-reported perception and physical measures such as proprioceptive drift (difference between perceived and actual body position) and electrophysiological muscle activity (Lenggenhager, Tadi, Metzinger, \& Blanke, 2007; Slater et al., 2008), lending credence to the convergent validity of self-report measures of body image alterations. Notably, however, the PI may be perceptually attributed to a single sensation or multiple concurrent sensations (i.e., arm extension alone, or arm extension accompanied by nose elongation), as the aforementioned internal model may be consolidated via any combination of mechanically plausible illusory occurrences. Thus, despite its titular moniker, there is a lack of convergence as to what perceptions necessarily constitute the PI.

PI measurement has evolved over time, with initial measurement via open-ended self-report of overall proprioceptive sensations and latency until sensations were experienced (see Supplementary Material, Table S1; Lackner, 1988). Later work aimed to quantify the intensity of the PI, having subjects estimate the length of finger displacement in centimeters and rate the "vividness" of the illusion using a 3-point Likert scale (Burrack \& Brugger, 2005). Recent research has taken a more granular approach, measuring discrete, illusory perceptual aberrations to better understand the overall phenomenon. Michael and Park (2016) compiled an 11-item Pinocchio Illusion Questionnaire (PIQ; Fig. 1) that included specific questions assessing commonly reported PI perceptions, seemingly unrelated sensations, and vibratory stimulation-induced somatosensory phenomena along a 5-point Likert scale.

Despite these advances, three primary issues remain in PI quantification. First, and most notably, the reliability of PI generation is currently unknown. Despite no reports of testretest reliability between administration sessions, it has become common practice to elicit the PI between four to six times per participant across differing conditions (i.e., arms/ vibration frequencies) and average scores across all sessions or only include sessions in which the PI was successfully elicited (Benson \& Park, 2019; Burrack \& Brugger, 2005;
Michael \& Park, 2016). Second, the PIQ contains questions that may be better attributed to vibratory stimulation than PI perception (e.g., finger tingling, arm pulsing). Benson and Park (2019) addressed this by proposing two PIQ subscales to distinguish between self-reported perceptual (Perceptual Aberration, PA) and somatosensory (Physical Sensation, PS) phenomena that result from illusion generation, though these subscales have not yet been empirically validated and the extent to which they measure perceptive phenomena is thereby still unknown. However, the PS subscale contains an item on temperature change and the PA subscale contains questions that are unrelated or even contradictory to proprioceptive afferent signals propagated by bicep brachii muscle spindle nerve signaling (i.e., nose widening, nose shrinking). Thus, these subscales are inconsistent with proprioceptive models of PI generation. Third, it remains unclear how such PIspecific items associate with well-validated measures of perceptual abnormalities. For example, the PIQ has been shown to be significantly correlated with the cognitive-perceptual factor (encompassing the subscales of Magical Thinking, Ideas of Reference, Unusual Perceptual Experiences, and Paranoid Ideation; Raine et al., 1994) of the Schizotypal Personality Questionnaire, but it is unknown whether this association is driven by specific SPQ subscales making up the broader factor (Benson \& Park, 2019; Michael \& Park, 2016). Taken together, proper validation and measures of internal consistency and construct validity are necessary to ensure that PIQ scores reflect perceptual aberrations specific to proprioceptive afferents, rather than general psychosomatic suggestibility or sensory vibratory stimulation.

The current study evaluates the test-retest reliability and construct validity of the PIQ and its proposed subscales, examines their convergent validities with two different perceptual measures, and elucidates the PIQ factor structure via a principal component analysis (PCA) in order to formulate empirically supported subscales. Because the PS subscale contains an item on temperature change and the PA subscale contains questions that are unrelated or even contradictory to specific proprioceptive afferent signals propagated by bicep brachii muscle spindle nerve signaling (i.e., nose widening, nose shrinking), it is hypothesized these subscales will not be supported by our results.

Based on previous studies reporting PI prevalence under differing parameters (Table 1), the PIQ is hypothesized to demonstrate good $(\rho>0.80)$ test-retest reliability and fair $(\alpha>0.70)$ internal consistency due to the inclusion of questions more related to somatic sensations rather than proprioceptive sensations previously reported during PI elicitation. It is also hypothesized that items related to movement or position (e.g., nose and arm elongation) would cluster in our PCA and explain the most PIQ variance and, finally, that our two aberrant proprioceptionrelated self-report measures would positively correlate with PIQ scores. Characterizing these psychometric properties of 
PI Score Sheet

A. Description of sensation(s):

B. Location of sensation(s):

(Not at all) 0--10--20--30--40--50--60--70--80--90-100 (Definitely)

1. I felt like my nose was pushing my finger forward.

2. My nose felt like it was becoming longer.

3. My nose felt like it was becoming wider.

4. My nose and my index finger felt disconnected from my hand and arm.

5. I felt a pulsation in my nose and/or index finger.

6. I felt a pulsation in my arm.

7. I felt "tingliness" in my nose and or index finger.

8. I felt "tingliness" in my arm.

9. My arm felt like it was extending forward.

10. My arm, index finger, or nose felt like it became warmer or colder.

11. My nose felt like it was becoming smaller.

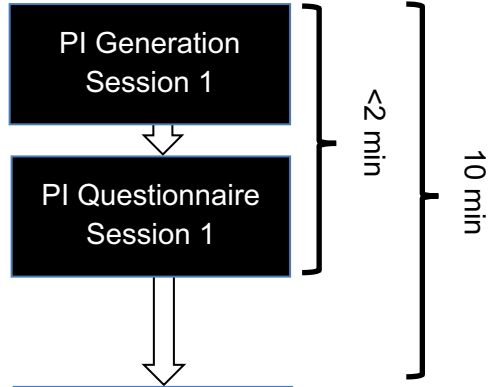

PI Generation

Session 2

PI Questionnaire Session 2

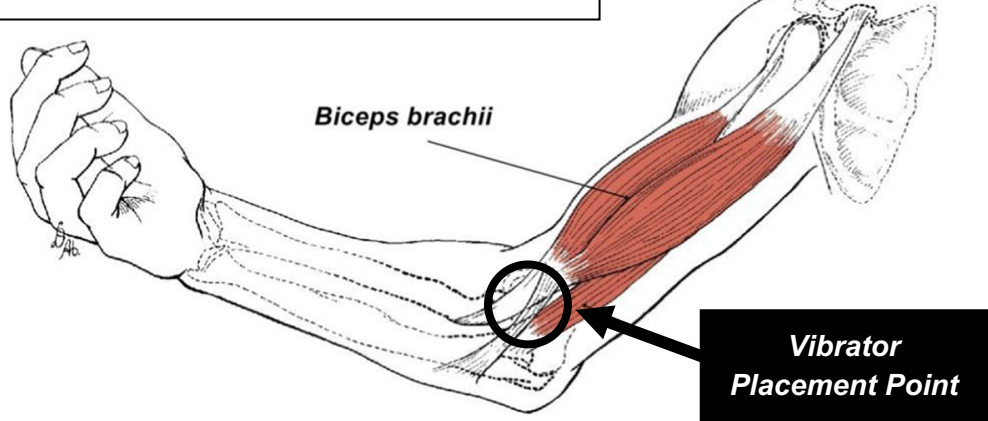

Fig. 1 Diagram illustrating location of vibratory stimulation and protocol of Pinocchio Illusion generation and Pinocchio Illusion Questionnaire (PIQ) administration. For the purposes of this study, questions A \& B were asked prior to items 1-11. PIQ adapted from Michael and Park

the PIQ is critical for understanding the proprioceptive constructs underlying the PI, which may further the application of this instrument to experimental and clinical questions.

\section{Methods}

\section{Participants}

All procedures were approved by an institutional review board. A total of 63 college undergraduates participated in this study and earned course credit towards a research experience requirement. Three participants were excluded for selfreported neurologic or psychiatric disorders and current illicit drug use, resulting in a final sample of 60 (34 women). The final sample was chosen as it is in accordance with current sample sizes for PI publications (i.e., Benson \& Park, 2019). The final sample had a mean age of 18.85 years (standard deviation $=1.33$ ) and was $65 \%$ White, $15 \%$ Asian, $6.7 \%$ Black, 5\% Hispanic/Latino, and $8.3 \%$ endorsed more than one racial/ethnic group or selected other racial/ethnic groups.
(2016). Graphic of arm and brachii reproduced with permissions from LifeART (c) 2019 Wolters Kluwer Health, Inc., Lippincott Williams \& Wilkins. All rights reserved

\section{Pinocchio Illusion (PI) induction and evaluation}

After providing written informed consent, participants were seated at a table and the first PI elicitation and measurement session began. There were 4-5 $\mathrm{min}$ between the end of Session 1 and the beginning of Session 2, such that the time between the start of Session 1 and the start of Session 2 was $10 \mathrm{~min}$ (Fig. 1). This pattern of back-to-back stimulation is consistent with prior literature; however, previous studies (c.f. Table 1) have targeted different arms and at differing vibration frequencies across stimulation sessions under the assumption that no carry-over effects occur in endorsement of the illusion. Participants were blindfolded and the left elbow was rested on the table. A folded hand towel was placed under the participant's elbow as a cushion to minimize discomfort and aid in arm muscle relaxation. The left bicep was flexed so that experimenters could locate the portion of the bicep brachii tendon nearest to the crook of the elbow. The head of a physiotherapy vibrator (Novafon Pro SK2, PZN 7105883) was then applied to this portion of the tendon. After vibrator placement, the forearm was raised so that the left index finger could touch the tip of the nose while the elbow remained on the table. Task 


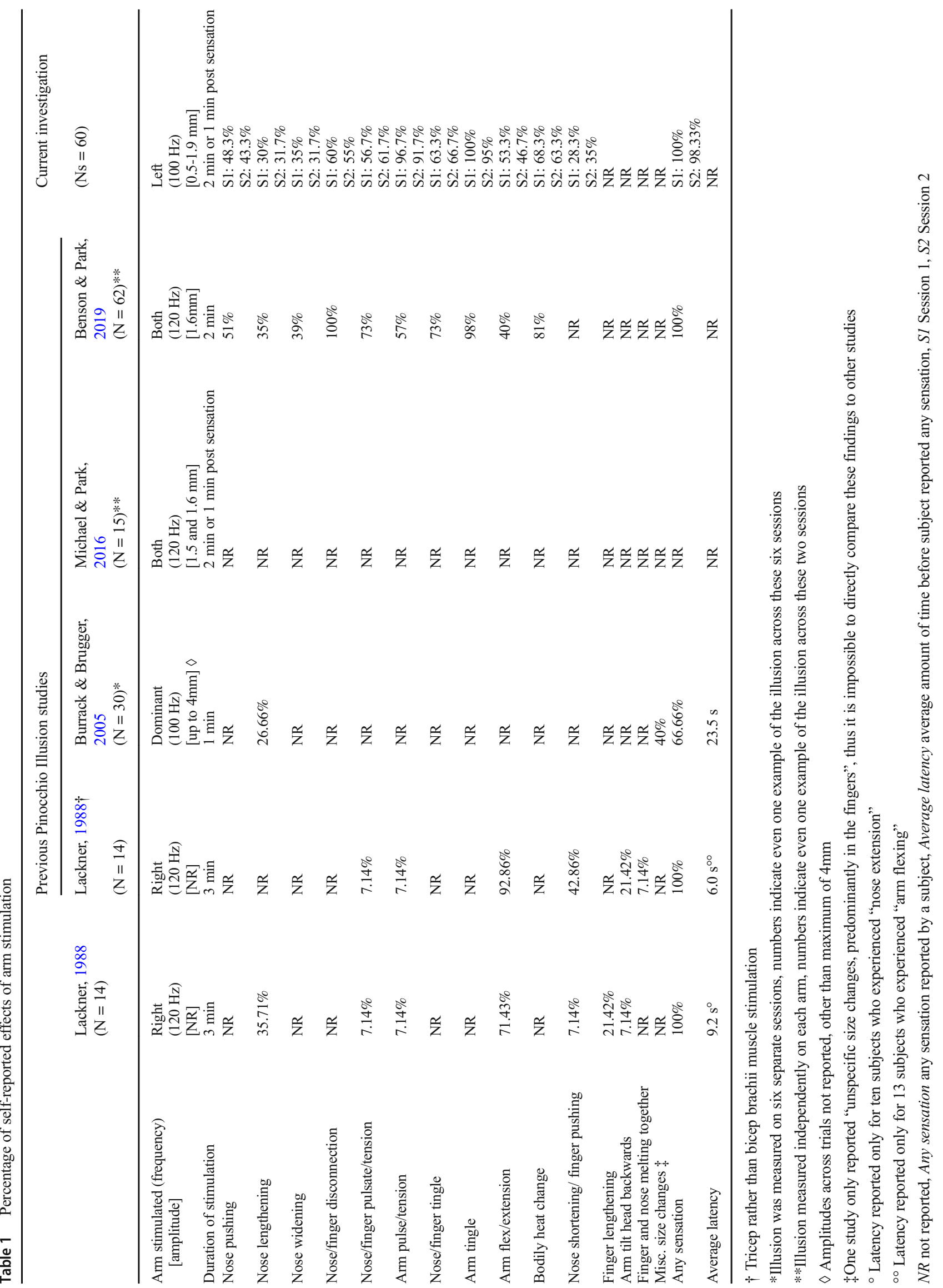


instructions were given before vibratory stimulation commenced. Following stimulation, the experimenter orally administered a modified version of the PIQ. Subjects were first given open-ended questions and then PIQ items. Each item could be answered on a scale from 0 to 100 , with 0 indicating that a phenomenon was not felt and 100 indicating that the phenomenon was felt with absolute certainty. Answers in between these two extremes indicate perception or sensation may have been perceived but with varying levels of doubt. For example, an answer of 50 to a given item would suggest that a participant reported feeling a phenomenon with a greater degree of certainty than one who answered 40 to the same item.

\section{Perceptual assessment}

Altered perceptions of self are frequently seen in psychosisspectrum disorders, with proprioceptive paradigms such as the PI and rubber hand illusion being noted to have enhanced elicitation in those with schizophrenia (Michael \& Park, 2016; Peled, Pressman, Geva, \& Modai, 2003; Thakkar, Nichols, McIntosh, \& Park, 2011). Accordingly, the Schizotypal Personality Questionnaire (SPQ; Raine, 1991) was administered -74 yes-no items in paper and pencil format - immediately following the second session to determine if PI perception was related to this external measure of clinically relevant perceptual aberrations. The SPQ has nine total subscales related to schizotypal personality disorders features, five of which were conceptually relevant to the current study due to their assessment of perceptual abnormalities expected to be related to perception of the PI illusion and previous correlations reported in the literature. These included Unusual Perceptual Experiences (e.g., When you look at a person, or yourself in a mirror, have you ever seen the face change right before your eyes?), Ideas of Reference (e.g., Do some people drop hints about you or say things with a double meaning?), Magical Thinking (e.g., "Have you had experiences with the supernatural?"), Paranoid Ideation (e.g., "I often feel that others have it In for me."), and No Close Friends (e.g., "I have little interest in getting to know other people."). Accordingly, a Bonferroni correction for five comparisons was performed for significance threshold of $p=$ 0.01 . An additional exploratory analysis was performed to determine convergent validity of the PI and self-reported interoceptive body awareness using the 32-item Multidimensional Assessment of Interoceptive Awareness (MAIA; Mehling et al., 2012). MAIA data were not collected from two subjects due to administration error. In addition, two subjects each left one MAIA item blank. These two missing datapoints were imputed via case-mean substitution according to their respective subscale means (FoxWasylyshyn \& El-Masri, 2005).

\section{Results}

\section{Descriptive statistics}

Data were analyzed using SPSS (25.0, IBM Corporation). Descriptive statistics can be found in Table 2. Due to the positively skewed distribution of the data, a $\log _{10}$ transform was applied for further analyses. In addition to reporting PIQ mean intensity ratings the number of subjects who reported any PI perception (i.e. non-zero endorsement of PI-specific items) are also reported in order to illustrate the number of subjects who reported experiencing the PI.

\section{Internal consistency and test-retest reliability}

Internal consistency was measured using Cronbach's alpha $(\alpha)$ calculated for all 11 items for both Session 1 (S1) and Session 2 (S2). The PIQ demonstrated good internal consistency for both sessions $\left(\alpha_{\mathrm{S} 1}=0.805, \alpha_{\mathrm{S} 2}=0.830\right)$. Good test-retest reliability was found using Spearman's rho $(\rho)$ correlations between S1 and $\mathrm{S} 2$ values for each individual PIQ item and the sum scores ( $\rho=0.730, p<0.001)$ (Table 2). Wilcoxson's sign-ranked tests then determined that the average PIQ S1 and S2 sum scores did not significantly differ, and only individual PIQ item average (\#11; "nose becoming smaller") was reported to have been more intensely experienced in $\mathrm{S} 2(\mathrm{Z}=-2.386, p=0.017)$. During the elicitation of open-ended reports of sensations immediately following stimulation but prior to PIQ administration only three subjects endorsed PI-like experiences following S1 (e.g., " ... as if arm moved," "felt like finger wanted to move away from nose"; "forearm/hand moving back and forth") and five subjects following S2 (e.g., "forearm getting longer"; "nose felt like it was being pulled forward..."; "disconnect between nose and finger"; for further information, see Supplementary Material, Fig. S2 and Table S5). The percentage of explicit endorsements (i.e., number of non-zero endorsements) of the PI-phenomenon was much more prevalent following the PIQ (Table 2).

\section{Principal components' analysis}

In order to discern the appropriateness of the two aforementioned PIQ subscales proposed by Benson and Park (2019), a two-component principal component analysis (PCA) with Varimax rotations was conducted (Table 3). Three- and fourcomponent PCAs were also evaluated, but their factor loadings were found to be unsatisfactory due to a lack of conceptual coherence and test-retest reliability (Table 3 ). Since the test-retest reliability of the PIQ was validated and previous studies have averaged PIQ scores over PI elicitation sessions, PCAs were carried out on the log-transformed averages of S1 and S2 scores. However, results from PCAs for individual sessions are included for transparency (Supplementary Material, Table S2). 
Table 2 Descriptive statistics and percent of non-zero endorsements for each Pinocchio Illusion Questionnaire (PIQ) items for Session 1 (S1) and Session 2 (S2)

\begin{tabular}{|c|c|c|c|c|c|c|c|c|}
\hline & \multicolumn{3}{|c|}{ Stimulation Session 1 (S1) } & \multicolumn{3}{|c|}{ Stimulation Session 2 (S2) } & \multirow[t]{2}{*}{ Spearman Rho $(\rho) \dagger$} & \multirow[t]{2}{*}{ Wilcoxon } \\
\hline & $\%$ Endorsed & Mean & $\mathrm{SD}$ & $\%$ Endorsed & Mean & SD & & \\
\hline Nose pushing finger & 48.33 & 17.17 & 23.08 & 43.33 & 17.50 & 26.78 & .635 & -.582 \\
\hline Nose lengthening & 30.00 & 6.83 & 13.21 & 31.67 & 11.17 & 22.63 & .750 & -1.139 \\
\hline Nose widening & 35.00 & 7.83 & 12.50 & 31.67 & 8.50 & 16.24 & .399 & -.215 \\
\hline Nose/finger disconnection & 60.00 & 22.83 & 22.41 & 55.00 & 20.50 & 24.18 & .640 & -.982 \\
\hline Nose/finger pulsate & 56.67 & 24.50 & 28.19 & 61.67 & 36.33 & 36.59 & .444 & -1.794 \\
\hline Arm pulse & 96.67 & 67.67 & 31.32 & 91.67 & 70.33 & 34.05 & .551 & -.787 \\
\hline Nose/finger tingle & 63.33 & 31.50 & 32.41 & 66.67 & 41.67 & 38.36 & .534 & -1.308 \\
\hline Arm tingle & 100.00 & 79.83 & 23.61 & 95.00 & 76.17 & 28.05 & .592 & -1.357 \\
\hline Arm extension & 53.33 & 26.83 & 30.50 & 46.67 & 25.00 & 32.39 & .618 & -1.029 \\
\hline Bodily heat change & 68.33 & 39.17 & 35.14 & 63.33 & 36.17 & 35.66 & .780 & -1.394 \\
\hline Nose shortening & 28.33 & 8.83 & 16.98 & 35.00 & 14.33 & 24.24 & .815 & $-2.386^{*}$ \\
\hline Total & 100 & 333.00 & 149.37 & 98.33 & 357.67 & 183.86 & .68 & -0.485 \\
\hline
\end{tabular}

$\dagger$ All Spearman's Rho $(\rho)$ correlations on raw scores significant $p<0.01$

$*=p<0.05$

Wilcoxon Wilcoxon signed-rank test on log-transformed data

The factor structure of S1\&S2 mean PIQ scores pointed to the multidimensional nature of the measure. The first principal component, which we titled "Illusory-Perceptions," consisted of items describing various proprioceptive and somatosensory perceptual aberrations that were related (e.g., nose elongation, arm extension), unrelated (e.g., nose widening, temperature changes), or contrary (e.g., nose shrinking) to existing theories and models of the PI (Table 3). Thus, items comprising Illusory-Perceptions were determined to illustrate overall sensitivity to a broad array of "Illusory-Perceptions" that are reported following PI generation, including but not specific to those underlying PI mechanics. The second principal component, named "Sensory-Vibration Byproducts," was comprised of items describing sensations attributed to vibratory stimulation (e.g., arm, finger, nose pulsing, and tingling) (Table 3). Thus, we propose that Sensory-Vibration Byproducts reflects general vibration sensitivity rather than sensitivity to bicep brachii stimulation in particular.

Spearman's rho $(\rho)$ was used to calculate inter-item correlations for these newly derived PCA components (i.e. Illusory-Perceptions and Sensory-Vibration Byproducts). It was found that all Illusory Perception items were positively

Table 3 Four-, three-, and two-factor principal component analysis (PCA) for average Pinocchio Illusion Questionnaire scores of Session 1 and Session 2 (S1 \& S2) item

\begin{tabular}{|c|c|c|c|c|c|c|c|c|c|c|c|c|c|c|}
\hline \multicolumn{15}{|c|}{ 4-, 3-, and 2-Factor PCAs (Average $\mathrm{S} 1+\mathrm{S} 2$ mean, $\log 10$ transformed } \\
\hline & & \multicolumn{11}{|c|}{ PIQ Items } & \multirow{2}{*}{$\begin{array}{c}\text { Internal } \\
\text { Consistency } \\
\text { (a) }\end{array}$} & \multirow{2}{*}{$\begin{array}{c}\text { Percent } \\
\text { Variance } \\
\text { Explained }\end{array}$} \\
\hline & & 1 & 2 & 3 & 4 & 5 & 6 & 7 & 8 & 9 & 10 & 11 & & \\
\hline \multirow{4}{*}{$\begin{array}{c}4 \\
\text { Factor }\end{array}$} & 1 & 468 & .673 & .850 & .449 & & & & & .660 & .328 & .875 & .818 & 26.98 \\
\hline & 2 & .559 & .423 & & & .783 & & .848 & & & & & .718 & 19.02 \\
\hline & 3 & & & & .579 & & & & .742 & & .758 & & .307 & 15.69 \\
\hline & 4 & -.335 & & & & .352 & .864 & & & & & & $N / A$ & 10.93 \\
\hline & & & & & & & & & & & & & & \\
\hline \multirow{3}{*}{$\begin{array}{c}3 \\
\text { Factor }\end{array}$} & 1 & .694 & .774 & .753 & .695 & & & .313 & & .707 & .563 & .744 & .856 & 33.35 \\
\hline & 2 & .384 & .309 & & & .809 & .403 & .824 & & & & & .749 & 16.68 \\
\hline & 3 & & & & & .323 & .693 & & .680 & & .513 & .324 & .337 & 13.65 \\
\hline & & & & & & & & & & & & & & \\
\hline \multirow{2}{*}{$\begin{array}{c}2 \\
\text { Factor }\end{array}$} & 1 & .705 & .785 & .769 & .709 & & & .346 & & .712 & .584 & .752 & .856 & 34.82 \\
\hline & 2 & & & & & .841 & .725 & .638 & .351 & & & & .620 & 18.43 \\
\hline
\end{tabular}

Internal consistency $=$ Cronbach's Alpha $(\alpha)$

Blank spaces indicate less than 0.03 factor loading

Data in italics indicate the items that load onto each factor 
correlated with one another (Supplementary Material, Table S3). Internal consistency $(\alpha)$ was also measured for each factor to determine consistent magnitude of PI item answers within individual factors. Internal consistency was found to be good for Illusory-Perceptions $(\alpha=0.863)$ and fair for Sensory-Vibration Byproducts $(\alpha=0.605)$.

\section{Convergent validity with self-report measures}

Spearman's rho with Bonferroni correction was used to determine associations between self-report measures and the PIQ due to the non-parametric distribution of the data. PIQ Illusory Perception subscale sum scores were significantly positively associated with the SPQ total score and, specifically, SPQ subscales of Unusual Perceptual Experiences $(\rho=0.370, p$ $=0.004)$, Ideas of Reference $(\rho=0.342, p=0.007)$, and No Close Friends $(\rho=0.334, p=0.009)$ (Table 4).

No statistically significant associations were found between MAIA and PIQ total and subscale scores (Supplementary Material, Table S5). Of note is the lack of significant association between the PIQ and the MAIA's Noticing and Non-Distracting subscales, which measure awareness of uncomfortable, comfortable, and neutral body sensations and one's tendency to pay attention to sensations of pain or discomfort (Mehling et al., 2012). This suggests individual variations in PI perception is not attributable to self-reported tactile sensitivity; a finding consistent with the previous literature (Benson \& Park, 2019). However, the lack of a significant association between the PIQ's Sensory-Vibratory Byproducts subscale and the MAIA may cast doubt on the extent to which the former measures sensitivity to vibratory stimulation resulting from PI generation.

\section{Discussion}

The current study investigated the validity and reliability of the PIQ in order to determine its appropriateness for research use and learn more about the construct it measures. Our results support good test-retest reliability and internal validity in measuring PI-associated body-image aberrations. However, they also point to a complexity of purported perceptual experiences resulting from PI generation that does not fully align with mechanistic theories or prior first-hand accounts of the PI as an illusory perception resulting solely from muscle spindle nerve ending signals (Burrack \& Brugger, 2005; Lackner, 1988). This is evidenced by three observations. First, perceptual phenomena both specific and non-specific to the PI exhibited considerable prevalence among our participants (Table 2). Self-report data and PIQ responses showed that participants endorsed these phenomena - which varied from nose elongation to nose shrinking to temperature changes - at similar frequencies.

Second, although our PCA effectively grouped items measuring Illusory-Perceptions and Sensory-Vibration Byproducts, it did not make such a distinction between theoretically supported/previously endorsed PI-specific and nonspecific perceptual phenomena - instead appearing to indiscriminately lump together all items describing IllusoryPerceptions. This was not resolved by increasing the number of components in the PCA (Table 3).

Third, items in the Illusory-Perceptions principal component, regardless of what specific perceptual phenomena they describe, were all positively correlated with one another (Supplementary Material, Table S3). This is particularly worrisome when examining how contradictory perceptions (e.g., nose elongation and nose shrinking) exhibit positive associations given that theoretical, proprioceptive models of PI generation are directional, with stimulation of the triceps brachii muscle resulting in sensations of arm flexion and feelings of the nose being pushed into or passing through the head (Lackner, 1988). This theoretical model and previous empirical evidence would preclude perceptions of these two movements from taking place simultaneously. Taken together, these suggest that Illusory Perception items and, to some extent, the PIQ as a whole may garner responses related to general proprioceptive anomalies as a result of demand characteristics

Table 4 Spearman's Rho correlations between the Pinocchio Illusion Questoinnaire (PIQ) and schizotypy

\begin{tabular}{|c|c|c|c|c|c|c|}
\hline & & \multicolumn{5}{|c|}{ PIQ correlation matrix with schizotypy } \\
\hline & & \multicolumn{5}{|l|}{ SPQ scores } \\
\hline & & Unusual Perceptual Experiences & Ideas of Reference & Magical Thinking & Paranoid Ideation & No Close Friends \\
\hline \multirow[t]{2}{*}{ PIQ } & Total & $.371 * *$ & $.314 *$ & .249 & .225 & $.317 *$ \\
\hline & Illusory perceptions & $.370^{* *}$ & $.342 * *$ & $.279 *$ & .251 & $.334 * *$ \\
\hline
\end{tabular}

$* *$ Significant at .01 (Bonferroni cutoff)

* Significant at 0.05 (uncorrected)

PIQ scores were taken from the average of S1 and S2 total scores and were log-transformed prior to analyses. The Schizotypal Personality Questionnaire (SPQ)'s No Close Friends subscale was included for analysis due to the observed relationship between the PIQ and subjective loneliness (cf., Michael \& Park, 2016) 
rather than genuine PI perception. This hypothesis is further bolstered by our findings that when asked open-endedly about any sensations only $5 \%$ of subjects (three of 60 participants) reported PI-related sensations at S1 and $8.3 \%$ (five of 60 participants) at S2 (Supplementary Material, Table S5), despite much greater endorsement following administration of each PIQ-specific item (Table 2). It is unlikely that the PIQ provided language that subjects were then able to use to describe PI perception, as open-ended reports of the PI were not more prevalent at S2 (i.e., after hearing the PIQ questions the first time) relative to $\mathrm{S} 1$.

The PA and PS subscales proposed by Benson and Park (2019) were mostly verified by our factor analyses, with the only discrepancy being the item measuring 'temperature change' grouping with questions describing PA rather than PS. This finding is consistent with our theory-driven conceptualization of perceptions in temperature change aligning more with Illusory-Perceptions rather than a Sensory-Vibration Byproduct, as bicep vibration should not trigger thermoreceptors (Schepers \& Ringkamp, 2010). While our factor analysis demonstrated that the Illusory-Perception and SensoryVibration Byproduct subscales are psychometrically sound, the current study provides no evidence that the IllusoryPerception subscale truly measures perceptual aberrations specific to the PI. Rather, this subscale may measure susceptibility to post-hoc reporting of illusory perceptions. While this result is not ideal for the quantification of the PI, it may be useful, instead, as a measure of somatosensory suggestibility.

As predicted, the PIQ's Illusory-Perception subscale was positively associated with the SPQ's Unusual Perceptual Experience subscale (Table 4). This convergent validity further contributes to the psychometric soundness of the PIQ as a measure of aberrant perceptual experiences. Moreover, the significant association between the PIQ's Illusory Perception subscale and SPQ Ideas of Reference subscale may reflect the importance of suggestibility or self-focused perceptions in the reporting of the PI illusion. Taken together, individuals who generally report experiencing more aberrant perceptions tend to also report experiencing the PI, a specific aberrant perception. In addition, these findings call into question whether the PIQ, and the PI more broadly, have been properly conceptualized. Scientific consensus must be achieved on what the PI construct is and what processes it includes (e.g., proprioceptive mismatch, suggestibility, and broad perceptual abnormalities). Suggestions for addressing this are elaborated below in Future directions. Other subscales composing the cognitiveperceptual factor originally identified to correlate with the PIQ such as Magical Thinking and Paranoid Ideation were not significantly associated following correction for multiple comparisons (Table 4). Conceptually, this finding aligns with the current framework as those subscales are not specific to self- or body-focused experiences or sensations. Finally, the positive association between the PIQ Illusory Perception subscale and the SPQ No Close Friends subscale builds upon previous research implicating the Social Deafferentation Hypothesis in illusion perception (Michael \& Park, 2016). This hypothesis posits that, as one's social network and amount of social interactions decreases, social cognition processes may functionally reorganize in response to their decreased utilization, resulting in perceptual anomalies (Hoffman, 2007).

\section{Limitations}

First, there are the unavoidable possibilities of priming, acquiescence bias, and/or demand characteristics due to the repeated administration of the PIQ and its items. However, experimenters were trained to deliver PIQ items with consistent inflection and intonation across participants so as to avoid confounding effects of demand characteristics. Second, this study used short inter-trial intervals to separate PI generation and measurement sessions while previous studies did not include breaks between these sessions as they did not stimulate the same arm with the same vibratory parameters (c.f. Table 1); thus, the effects of the inter-trial interval on PIQ responses requires further study. A longer break duration (i.e., several hours) may be preferable to several minutes in order to reduce carry-over effects. Third, unstandardized elements of the PI-elicitation protocol which vary among the literature and our study may affect the success of illusion generation. For example, the minimum pressure with which vibratory stimulation could activate bicep muscle spindle nerve endings has not been reported. Therefore, it is possible that failure to generate the illusion in our participants could result from inadequate muscle stimulation. Finally, the sample size $(n=60)$ is modest for a PCA, but sufficient to evaluate a bi-factor model. Further research should confirm these findings with a larger sample.

\section{Future directions}

As alluded to in this study, a major downfall of the PIQ is the inability for a participant to access and accurately convey subjective perceptual information and for the measure to clearly capture it. Future research into the PI might be improved by implementing more objective measures to directly evaluate such perceptions, without requiring mental distance estimation. The following may resolve this issue: Participants begin by placing both hands parallel, and in mirrored finger positions, with both elbows on the table prior to stimulation. The pointer finger on the non-PI hand would not be touching the nose/face, but rather floating freely in mid-air. Following arm stimulation, the participant could be asked to align the hand on the unstimulated arm with the hand that is touching the nose. The forward distance between fingertip locations would provide a perceived distance of arm extension/nose elongation 
regardless of perceived bodily alteration. This recommendation negates the subjective nature of any form of questionnaire and provides an objective, quantifiable distance of the illusion that does not require the subject to mentally estimate distances in centimeters or inches. Moreover, the strength of the perception could then be assessed on a continuum (i.e., distance), rather than on a dichotomous (positive or negative endorsement) or ordinal (i.e., 1-10 self-report) scale. For more stringent methods, sophisticated arm rest apparatuses have been implemented for task consistency (Burrack \& Brugger, 2005). Such objective measures could be used to better validate selfreport measures or, perhaps, might prove sufficient and less burdensome to researchers and participants without need for subjective assessments.

Additionally, future research should seek to better develop self-report measures for understanding the perception of the PI, though this direction is not recommended over, or without the inclusion of, the establishment of objective tools to determine the validity of the phenomenon. As illuminated by the current study, the PIQ in its current form may be tap into other constructs, such as suggestibility and trait-level atypical perceptual experiences, which may obscure the perceptual illusion of interest. Further construct development will be key in the continued elicitation and assessment of the PI in research. Important steps in this process would include collecting data from large samples and determining which questions differentiate successful PI generation across different subjects and across stimulation sessions within a subject.

Further research for improving the PIQ is encouraged in the following ways. (1) The addition of questions completely disparate to the PI (e.g., "my ears felt like they floated higher on my head," "leg vibration ipsilateral to stimulated arm") may be added to the PIQ to better determine individual susceptibility to over-reporting completely irrelevant bodily sensations. Such questions may aid in determining whether subjects are generally overreporting or merely misattributing perceived sensations according to the questionnaire items available (e.g., describing flexing sensation as "heating"). (2) After several additional PI-related and PI-unrelated items have been conceptualized, they may all be administered to subjects who have demonstrated susceptibility to the PI through the afore suggested objective measurement or free response endorsement. This method allows for the questionnaire to discern which specific items correspond with more rigorous endorsements of the perceived illusion. It may be the case that the PIQ in its current form truly describes the PI, but no published studies include information on subjects' free responses following stimulation before PIQ administration (Benson \& Park, 2019; Michael \& Park, 2016). (3) Using item response theory-based analyses could determine which questions differentiate successful PI generation across different subjects as well as across stimulation sessions within a subject. (4) Finally, a variety of other perceptual and sensorimotor tasks may be used to validate assessments of the PI and more carefully elucidate the construct of "bodily plasticity" that purportedly underlies the PI experience.

\section{Conclusions}

Though previous studies have used PIQ as an indicator of PI susceptibility, our results suggest a disconnect between susceptibility and phenomenology. Despite displaying acceptable psychometric properties (test-retest reliability, internal consistency, and convergent validity), the construct validity of the PIQ in measuring PI phenomena remains unclear. Our results suggest that the PIQ and the Illusory-Perceptions subscale quantify general endorsement of perceptual experiences that cannot be solely attributed to bicep brachii vibration and may reflect a general susceptibility to perceptual anomalies. Accordingly, there is a need to further refine PIQ items so that they more directly and specifically pertain to mechanistically supported PI-related illusory experiences. Moreover, the current work points to the broader importance of ensuring target specificity in measurement tools used to quantify phenomenologically experienced changes during the perception of bodily illusions.

Acknowledgements The authors would like to acknowledge Richard Viken for his helpful feedback on early drafts of this manuscript.

Funding This research was supported in part by Indiana University Hutton Honors Undergraduate Research Grant (JC), Indiana CTSI Predoctoral Grant [UL1TR001108 \& TL1TR002531 (JRP; ABM)], and the National Institute of Mental Health [T32MH103213 (JRP; ABM)], [R01 MH074983 (WPH)]. The content is solely the responsibility of the authors and does not necessarily represent the official views of the NIMH.

\section{References}

Benson, T. L., \& Park, S. (2019). Increased plasticity of bodily selfexperience in individuals who may carry latent liability for schizophrenia. Schizophrenia Research, 207, 58-62.

Burrack, A., \& Brugger, P. (2005). Individual differences in susceptibility to experimentally induced phantom sensations. Body Image, 2(3), 307-313.

Fox-Wasylyshyn, S. M., \& El-Masri, M. M. (2005). Handling missing data in self-report measures. Research in Nursing \& Health, 28(6), 488-495.

Goodwin, G. M., McCloskey, D. I., \& Matthews, P. B. C. (1972). The contribution of muscle afferents to keslesthesia shown by vibration induced illusions of movement and by the effects of paralysing joint afferents. Brain, 95(4), 705-748.

Hoffman, R.E. (2007). A social deafferenation hypothesis for induction of active schizophrenia. Schizophrenia Bulletin, 33(5), 1066-1070. 
Lackner, J. R. (1988). Some proprioceptive influences on the perceptual representation of body shape and orientation. Brain, 111(2), 281297.

Lackner, J. R., \& Taublieb, A. B. (1984). Influence of vision on vibrationinduced illusions of limb movement. Experimental Neurology, 85(1), 97-106.

Lenggenhager, B., Tadi, T., Metzinger, T., \& Blanke, O. (2007). Video Ergo Sum: Manipulating Bodily Self-Consciousness. Science, 317(5841), 1096-1099.

McCloskey, D. I. (1973). Differences between the senses of movement and position shown by the effects of loading and vibration of muscles in man. Brain Research, 61, 119-131.

Mehling, W.E., Price, C., Daubenmier, J.J., Acree, M., Bartmess, E., \& Stewart A. (2012). The Multidimensional Assessment of Interoceptive Awareness (MAIA). PLoS ONE, 7(11), e48230.

Michael, J., \& Park, S. (2016). Anomalous bodily experiences and perceived social isolation in schizophrenia: an extension of the social deafferentation hypothesis. Schizophrenia Research, 176(2), 392397.

Peled, A., Pressman, A., Geva, A.B. \& Modai, I. (2003). Somatosensory evoked potentials during a rubber-hand illusion in schizophrenia. Schizophrenia Research, 64(2-3), 157-63

Proske, U., \& Gandevia, S. C. (2012). The proprioceptive senses: Their roles in signaling body shape, body position and movement, and muscle force. Physiological Reviews, 92(4), 1651-1697.

Raine, A. (1991). The SPQ: A Scale for Assessment of Schizotypal Personality Based on DSM-III-R Criteria. Schizophrenia Bulletin, 17(4), 555-64.
Raine, A., Reynolds, C., Lencz, T., Scerbo, A., Triphon, N., \& Kim, D. (1994). Cognitive-perceptual, Interpersonal, and Disorganized Features of Schizotypal Personality. Schizophrenia Bulletin, 20(1), 191-201.

Roll, J., \& Vedel, J. (1982). Kinaesthetic role of muscle afferents in man, studied by tendon vibration and microneurography. Experimental Brain Research, 47(2).

Schepers, R. J., \& Ringkamp, M. (2010). Thermoreceptors and thermosensitive afferents. Neuroscience \& Biobehavioral Reviews, 34(2), 177-184.

Slater, M., Pérez Marcos, D., Ehrsson, H., \& Sanchez-Vives, M. V. (2008). Towards a digital body: the virtual arm illusion. Frontiers in Human Neuroscience, 2, 6.

Thakkar, K.N., Nichols, H.S., McIntosh, L.G. \& Park, S. (2011). Disturbances in Body Ownership in Schizophrenia: Evidence from the Rubber Hand Illusion and Case Study of a Spontaneous Out-ofBody Experience. PLOS One, 6(10), e27089.

Open Practices Statement All of the data for the experiment reported in this publication is available and the experiment was not preregistered.

Publisher's note Springer Nature remains neutral with regard to jurisdictional claims in published maps and institutional affiliations. 